Trauma Berufskrankh 2008 10

[Suppl 3]:341-350

DOI 10.1007/s10039-007-1332-2

Online publiziert: 23. Januar 2008

(c) Springer Medizin Verlag 2008

\author{
M. Behrens \\ Berufsgenossenschaft Chemie, Köln
}

\title{
Ergebnisse des Projekts „Medizinische Rehabilitation“
}

sicherungsträger der öffentlichen Hand teilnahmen, abgeschlossen. Über einige seiner Ergebnisse soll im Folgenden auszugsweise berichtet werden.

\section{Projektansatz}

\section{Grundlegende Überlegungen - Projektziele}

Das Projekt „Medizinische Rehabilitation" diente in erster Linie einem Vergleich der Ergebnisse, die die beteiligten Träger durch ihre Heilverfahrenssteuerung erzielen. Durch Analyse der verwaltungsseitigen Heilverfahrenssteuerung sollten Best Practices („Lernen vom Besten“) gefunden werden, um die aus dem Benchmarking sprechenden Optimierungspotenziale für die Teilnehmer realisierbar zu machen [1]. Zudem sollte die Wirksamkeit von Steuerungsmechanismen der Verwaltung durch die Bestätigung von Hypothesen zur Heilverfahrenssteuerung belegt werden.

Beginnend mit der Eingangssachbearbeitung stellt sich die Frage, welche validierte Diagnose vorliegt und ob der Versicherte in ein zum Verletzungsartenverfahren zugelassenes Krankenhaus zu verlegen ist. Auch drohende oder bereits eingetretene Komplikationen des Heilverlaufs erfordern die Behandlung Unfallverletzter in Kompetenzzentren. Ein modernes Reha-Management setzt schließlich eine Planung, Ausführung und Steuerung der individuellen Rehabilitation der Versicherten anhand eines Rehabilitationsplans unter partnerschaftlicher Einbindung der behandelnden Ärzte, Therapeuten und der Versicherten mit ihren Angehörigen sowie - bei Einverständnis des Ver- sicherten - auch des Arbeitgebers voraus. Durch die Steuerung nach Heilverfahrensendterminen und Kooperationen mit externen Beratern werden zusätzlich zu diesen Mechanismen eine gesteigerte Effektivität und Effizienz bei der Rehabilitation der Versicherten angestrebt. Offen ist jedoch, in welchem Umfang sich diese Bemühungen auswirken auf:

- die Dauer der Arbeitsunfähigkeit,

- die Kosten der Heilbehandlung,

- das Heilergebnis und

- die Frage, ob und in welcher Höhe eine Verletztenrente zu zahlen ist.

Durch die Auswertung von Akten anhand normierter Fragebögen sollten deshalb Rückschlüsse darauf gezogen werden, welche Weichenstellungen in der Heilbehandlung und der Sachbearbeitung das Ergebnis des Heilverfahrens positiv beeinflussen.

\section{Ein- und Ausschlusskriterien}

Ausgangspunkt des Benchmarkings war ein Vergleich der Heilverfahrensergebnisse anhand vordefinierter Unfallverletzungen, die nach vorgegebenen Kriterien vorgesichtet und ausgewählt wurden, da die Verschlüsselung der Fälle kein zuverlässiges Kriterium für die Auswahl vergleichbarer Fälle darstellte - zum einen erwies sie sich als zu unspezifisch, zudem erfolgte sie in vielen Fällen nicht mit der gebotenen Genauigkeit. Auf diese Weise konnte sichergestellt werden, dass es sich um Verletzungen mit validierter Diagnose und vergleichbarer Schwere handelte. Damit diese für das Benchmarking tauglich waren, mussten sie mit ausreichender Fallzahl bei allen Teilnehmern als Versi-
Im Jahr 2006 wurde das Projekt, an dem 7 Berufsgenossenschaften und 2 Unfallver- 
cherungsfälle vorhanden sein. Zusätzlich musste es sich um Verletzungen handeln, die aufgrund ihrer Komplexität erfahrungsgemäß ein überdurchschnittliches Komplikations- und Steuerungspotenzial aufweisen, sodass - je nach Erfolg der Steuerung - erkennbare Unterschiede im Heilergebnis erwartet werden konnten.

Nach einer Vorauswertung legte sich die Arbeitsgruppe entsprechend der oben genannten Kriterien auf 3 Verletzungen fest, die als Benchmarkingobjekte einbezogen wurden:

- Fraktur des Fersenbeins

- Fraktur des oberen Sprunggelenks

- Ruptur des vorderen Kreuzbands

Nach Beratung durch die am Projekt beteiligten Unfallchirurgen wurden Ausschlusskriterien formuliert, durch deren Anwendung ein Kollektiv vergleichbarer Fersenbeinfrakturen für das Benchmarking sichergestellt werden konnte, sodass eine nachträgliche Klassifikation der einzelnen Fälle nicht erforderlich wurde. Für die Fraktur des oberen Sprunggelenks (Weber-C-Fraktur) und die Zerreißung bzw. den Teilriss des vorderen Kreuzbands im Knie wurden entsprechende Ausschlusskriterien vereinbart.

Der Umstand, dass sich aufgrund der Ausschlusskriterien einige Teilnehmer beim Benchmarking nur mit verhältnismäßig kleinen Fallzahlen beteiligen konnten, musste aufgrund des Vorrangs, nur vergleichbare Verletzungen in ihrer Heilverfahrenssteuerung zu beurteilen, in Kauf genommen werden und beeinträchtigte die Aussagekraft des Benchmarkings nach übereinstimmender Auffassung nicht. Dabei ist zu erwähnen, dass durch die Einbindung von externem Sachverstand aus den Bereichen Unfallchirurgie, Betriebswirtschaft und Statistik eine interdisziplinäre Bündelung der benötigten Kompetenzen die Gewähr dafür bot, die in der Projektgruppe tätigen Vertreter der Benchmarkingteilnehmer methodisch zu unterstützen. Diese unverzichtbare Beratung konnte insbesondere durch die Arbeit der Forschungsstelle Benchmarking an der Justus-Liebig-Universität Gießen und mehrere Oberärzte von berufsgenossenschaftlichen Unfallkliniken sichergestellt werden.
Das Benchmarking der Heilverfahrensergebnisse wurde jeweils getrennt nach obigen 3 Verletzungen durchgeführt. Schon nach der Vorauswertung war aus der Datenlage erkennbar, dass die Erhöhung der Betrachtungsschärfe durch strikte Beschränkung auf vergleichbare Verletzungen unbedingten Vorrang vor der Analyse hoher Fallkontingente mit in sich inhomogenen Verletzungsbildern haben muss, da ansonsten keine Aussagen zu Zusammenhängen zwischen Maßnahmen der Heilverfahrenssteuerung und deren Ergebnisse möglich sind.

\section{Kategorisierung der Fälle}

Hierfür wurde ein Benchmarkingportfolio gebildet. Dabei wurde auf die erreichte Rehabilitation der Versicherten abgestellt. Es wurden 4 verschiedene Cluster gebildet. Hauptkriterium für die Bildung des Portfolios stellte die Größe „Minderung der Erwerbsfähigkeit“ dar. Weitere Benchmarkingkriterien (Erfolgsfaktoren) für die 3 oben genannten Verletzungen waren die Dauer und Kosten des Heilverfahrens sowie der Erhalt des Arbeitsplatzes.

In Cluster 1 wurden die Fälle eingruppiert, bei denen eine vollständige Wiederherstellung gelang. In Cluster 2-4 wurden nach Schwere gestaffelt unterschiedliche Grade von verbliebenen Unfallfolgen abgebildet. In den einzelnen Clustern wurde auf diese Weise ein jeweils homogenisiertes Fallkontingent verglichen. Innerhalb der Cluster wurde mit Hilfe der Erfolgsfaktoren eine Reihenfolge der einzelnen Teilnehmer ermittelt.

Die einzelnen Cluster im Benchmarkingportfolio wurden wie folgt definiert:

Cluster 1: „Keine Verletzungsfolgen und Dauerschäden“. Ergebnis des Rehabilitationsprozesses ist die vollständige, folgenlose Ausheilung der Verletzung.

Cluster 2: „Geringfügige Verletzungsfolgen mit Dauerschaden ohne dauerhaften Rentenanspruch“. Innerhalb dieses Clusters wurden Fälle untersucht, bei denen am Ende der Rehabilitation geringe Unfallfolgen zurückblieben, die keinen Anspruch auf eine Verletztenrente auf unbestimmte Zeit begründeten. Dies kann bedeuten:
- dass die Minderung der Erwerbsfähigkeit dauerhaft unter $20 \%$ lag,

- dass nur eine Rente auf Zeit gezahlt wurde, in der eine Minderung der Erwerbsfähigkeit von $20 \%$ oder mehr vorlag bzw.

- dass eine Gesamtvergütung gezahlt wurde für eine Zeit, in der eine Minderung der Erwerbsfähigkeit von $20 \%$ oder mehr angenommen wurde.

Cluster 3: „Bleibende Verletzungsfolgen mit Dauerschaden sowie Rentenanspruch“. Hier wurden alle Fälle betrachtet, in denen eine Rente auf unbestimmte Zeit nach einer MdE von 20\% verblieb.

Cluster 4: „Bleibende Verletzungsfolgen mit erheblichem Dauerschaden". Alle Fälle, in denen ein Anspruch auf eine Verletztenrente auf unbestimmte Zeit mit einer MdE von über 20\% verblieb, wurden in Cluster 4 untersucht. Diese Fälle stellten im Zusammenhang mit den betrachteten Benchmarkingobjekten Worst-Case-Szenarien dar und wurden deshalb noch einmal getrennt von den anderen Dauerrentenfällen ausgewertet.

\section{Ergebnisse Datenvergleich}

\section{Steuerungseffekte bei frühzeitigem Vorliegen einer validierten Diagnose}

Eine Grundvoraussetzung für die verletzungsspezifische Steuerung des Heilverfahrens unmittelbar nach einem Arbeitsunfall ist das zeitnahe Vorliegen der validierten Diagnose. Es ist schlechthin nicht möglich, ein Steuerungs- und Überwachungssystem erfolgreich durchzuführen, wenn dem Versicherungsträger nicht rechtzeitig und ausreichend Kenntnis über die Ausgangssituation (Erstbefund) und den Heilverlauf gegeben wird [13].

Die medizinische Rehabilitation beginnt bereits unmittelbar nach dem Eintritt eines Arbeitsunfalls. Die in der Akutbehandlung getroffenen Weichenstellungen können einen erheblichen, entscheidenden Einfluss auf den Erfolg der Heilbehandlung haben [5]. Der ärztliche Erstbericht ist neben der vom Unternehmer zu erstattenden Unfallanzeige daher einer 
der wichtigsten Ersteingänge in der täglichen Sachbearbeitung. Durch ihn wird das berufsgenossenschaftliche Verwaltungsverfahren eingeleitet, da der Unfallversicherungsträger in der Regel originär durch den Erstbericht des Durchgangsarztes (Durchgangsarztbericht, D-ArztBericht) Kenntnis vom Unfall erhält.

Im Ärztevertrag ist die Erstattung des Durchgangsarztberichts in $\$ 27$ Abs. 2 festgelegt. Hiernach ist der erstbehandelnde Durchgangsarzt verpflichtet, den Verunfallten ärztlicherseits zu versorgen, zu untersuchen und den UV-Träger unverzüglich - möglichst noch am Unfalltag - über das Ergebnis der Untersuchung zu informieren. Dies gilt auch bei Wiedererkrankungen (\$27 Abs. 3 ÄV).

Bei der erstmaligen Konsultation durch den Unfallverletzten ist der Durchgangsarzt verpflichtet, die ärztliche Erstversorgung vorzunehmen, die allgemeine oder besondere Heilbehandlung einzuleiten und den zugrunde liegenden Sachverhalt aufzunehmen. Hierzu muss er sich im Rahmen der Untersuchung insbesondere mit der Frage befassen, ob überhaupt ein Arbeitsunfall vorliegt und die hierzu festgestellten Fakten dokumentieren. Der Durchgangsarzt muss in diesem Zusammenhang auch Zweifel über die Diagnose oder den ursächlichen Zusammenhang eines Leidens mit den vom Unfallverletzten geschilderten Beschwerden klären, was z. B. insbesondere bei Verletzungen im Bereich des Kniegelenks von sehr großer Relevanz ist.

Sofern vom behandelnden Durchgangsarzt die Kausalität der geklagten Verletzung und die Zuständigkeit des Unfallversicherungsträgers zweifelsfrei bejaht werden, wird er entsprechend den Vorgaben des Ärztevertrags die allgemeine oder besondere Heilbehandlung einleiten.

Für die weitere Betrachtung wird von der Einleitung besonderer Heilbehandlung ausgegangen, da dieses Vorgehen für die im Rahmen des Projekts untersuchten Verletzungen als obligat betrachtet werden kann.

Die umgehende Erstattung des Durchgangsarztberichts ist einer der essenziellen Bausteine der berufsgenossenschaftlichen Heilverfahrenssteuerung. Es liegt auf der Hand, dass ein frühzeitiger Eingang desselben der Berufsgenossenschaft eine zeit-

Trauma Berufskrankh 2008 10 [Suppl 3]:341-350 DOI 10.1007/s10039-007-1332-2

(c) Springer Medizin Verlag 2008

\section{Behrens \\ Ergebnisse des Projekts „Medizinische Rehabilitation“}

\section{Zusammenfassung}

Der Benchmarking-Club der Träger der gesetzlichen Unfallversicherung führte das erste trägerübergreifende Benchmarking zum Thema "Medizinische Rehabilitation" durch. Als Benchmarkingobjekte dienten Frakturen des Fersenbeins bzw. des oberen Sprunggelenks und Rupturen des vorderen Kreuzbands. Anhand der Projektergebnisse können als Best Practice formuliert werden: Es sollte frühestmöglich auf die Validierung der Diagnose hingewirkt werden. Schwere Verletzungen sowie solche mit Nebendiagnosen oder Komplikationen sollten zeitnah in einem Kompetenzzentrum behandelt werden. Bei Verzögerung des Heilverfahrens müssen unverzüglich die Berufshilfe bzw. der Reha-Manager eingeschaltet werden. Insbesondere bei po- tenziell retardierten Heilverläufen ist ein frühzeitiges Reha-Management indiziert. Trotz der Vergleichbarkeit der analysierten Unfallverletzungen ergaben sich erhebliche Unterschiede zwischen den Teilnehmern im Hinblick auf die Erfolgsfaktoren Dauer der Arbeitsunfähigkeit, Kosten der Rehabiliation, Erhaltung des Arbeitsplatzes und verbleibende Minderung der Erwerbsfähigkeit. Zusammenfassend gesehen ist Benchmarking ein Weg, Optimierungspotenziale für die Heilverfahrenssteuerung aufzuzeigen.

\section{Schlüsselwörter}

Benchmarking-Club - Medizinische Rehabilitation · Fersenbein · Oberes Sprunggelenk · Vorderes Kreuzband

\section{Results of the project "Medical Rehabilitation"}

\section{Abstract}

The Benchmarking Club of the statutory accident insurance providers carried out the first interdisciplinary benchmarking on the topic of "medical rehabilitation." Calcaneal or ankle joint fractures and ruptures of the anterior cruciate ligament served as the benchmarking objects. Based on the project results, the best practice can be formulated as follows: efforts should be made to validate the diagnosis as early as possible. Serious injuries as well as those with additional diagnoses or complications should be treated promptly in a trauma center affiliated with the accident insurance association. If therapy is delayed, the agency for occupational assistance or the rehabilitation manager must be called in immediately. In the case of a potentially delayed healing process, early rehabilitation management is indicated. Despite the comparability of the accident injuries analyzed, considerable differences between the participants were apparent. In summary, benchmarking is a way to identify optimization potentials for managing therapy. However, to what extent it can contribute to a more goal-oriented use of resources and implementation of efficiency potentials is at present still completely open.

\section{Keywords}

Benchmarking Club $\cdot$ Medical rehabilitation . Calcaneus · Ankle joint .

Anterior cruciate ligament 


\section{Benchmarking in der Gesetzlichen Unfallversicherung}

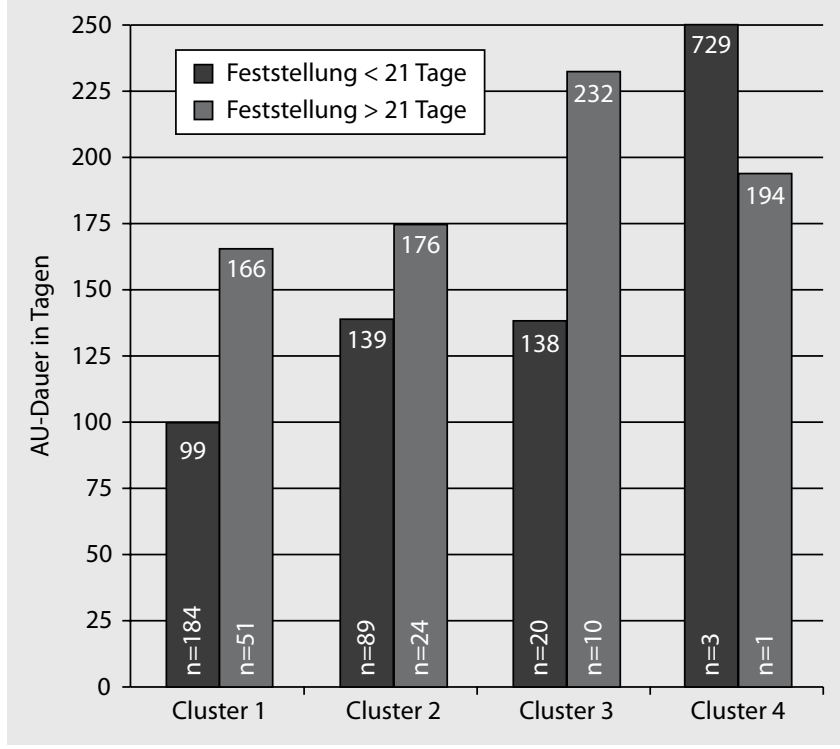

Abb. $1 \varangle$ Feststellung der validierten Diagnose bei Ruptur des vorderen Kreuzbands nahe und umgehende Intervention ermöglicht. Im Rahmen des Projekts war zu diskutieren, ob sich diese theoretische Annahme anhand der untersuchten Fälle belegen lässt.

\section{Resultate}

Die Annahme, dass die frühzeitige Kenntnis der (validierten) Diagnose eine bessere Heilverfahrenssteuerung ermöglicht, hat sich bei der Auswertung der Fälle, in denen als Unfallverletzung eine Ruptur der vorderen Kreuzbänder vorlag, bestätigt. Bei der validierten Diagnose dieser Verletzung zeigte sich mit Ausnahme von Cluster 4 in allen Clustern eine deutliche Zunahme der AU-Dauer, wenn die Validierung der Diagnose mehr als 21 Tage in Anspruch nahm, was für eine Bestätigung der Hypothese spricht (• Abb. 1).

\section{Resümee}

Als Best Practice kann das frühestmögliche Hinwirken auf die Validierung der Diagnose formuliert werden.

\section{Behandlung in Kompetenzzentren}

Die frühzeitige Einweisung oder Verlegung eines Unfallverletzten in ein Kompetenzzentrum wurde als weitere Steuerungsmöglichkeit der Verwaltung im Heilverfahren angesehen, von der in unterschiedlichem Ausmaß Gebrauch gemacht wird. Es stellte sich damit die Frage, ob eine medizinisch besonders fundierte Versorgung ihren Ausdruck in insgesamt niedrigeren Gesamtfallkosten und einer kürzeren Arbeitsunfähigkeitsdauer findet.

Betrachtet man das Verhältnis der Fallzahlen, in denen in der ersten Behandlungsphase nach einem Unfall einerseits BG-Unfallkliniken und andererseits zum Verletzungsartenverfahren (VAV) zugelassene Krankenhäuser als Leistungserbringer der medizinischen Rehabilitation in Anspruch genommen wurden, vermitteln die absoluten Zahlen auf den ersten Blick den Eindruck, dass nur ein sehr geringer Teil der überdurchschnittlich schweren Verletzungen, die ins Benchmarking einbezogen wurden, in eine BGUnfallklinik gesteuert und dort behandelt wird. Bei der Interpretation dieser Zahlen ist jedoch zu bedenken, dass den bundesweit rund 600 zum VAV zugelassenen Krankenhäusern lediglich 9 BG-Unfallkliniken gegenüberstehen. Vor diesem Hintergrund relativiert sich das scheinbare Ungleichgewicht bezüglich der Beteiligung an der Erstbehandlung.

Dabei ist anzumerken, dass die Steuerung eines unfallverletzten Versicherten in eine BG-Unfallklinik nicht zum vordergründigen Ziel haben kann, die stationären Kosten möglichst niedrig zu halten. Da durch die Verlegung in eine BGKlinik eine optimale Heilbehandlung mit allen geeigneten Mitteln (Maximalversorgung) bewirkt wird, können hierdurch initial auch höhere Kosten entstehen, als dies in anderen Krankenhäusern der Fall wäre. Durch diese Investition können jedoch retrospektiv betrachtet die Gesamtkosten des Falls vergleichsweise niedrig gehalten werden, da stets in die Betrachtung einbezogen werden muss, dass die Unfallversicherungsträger nicht nur die Kosten der Heilbehandlung selbst, sondern auch die Aufwendungen für vermeidbare Folgebehandlungen, Revisionsoperationen, Verletztengeldzahlungen und schlussendlich Verletztenrenten zu tragen haben. Diese Kosten, die aus einer unzureichenden medizinischen Rehabilitation in fachlich weniger versierten Krankenhäusern entstehen können, machen einen vielfach höheren Anteil der finanziellen Belastung bei den Unfallversicherungsträgern aus als die Kosten für die stationäre Heilbehandlung. Vorrangiges Ziel muss bei dieser Gesamtbetrachtung also die Nachhaltigkeit der Ergebnisqualität sein.

Bereits vor diesem Hintergrund erweist sich die frühestmögliche Einbeziehung der unfallmedizinischen Kompetenz von BG-Unfallkliniken in die Steuerung des Heilverfahrens als Vorteil sowohl für den von einer Verletzung betroffenen Versicherten als auch für den Kostenträger, wenn es gelingt, die zahlenmäßig relativ wenigen Fälle im Verhältnis zur Gesamtzahl der Unfälle zeitnah zu erkennen, die einen verlängerten oder kostenintensiven Verlauf des Heilverfahrens erwarten lassen. Entsprechende Projekte verschiedener Berufsgenossenschaften zur Intensivierung der Kooperation mit BG-Unfallkliniken während der Heilverfahrenssteuerung weisen mit ihren Einsparungserfolgen darauf hin [6].

\section{Resultate}

Im Rahmen der Analyse stellte sich heraus, dass in den BG-Unfallkliniken bereits bei der Erstversorgung eher Fälle mit schwierigen Verlaufsformen bzw. Nebendiagnosen behandelt werden. Es kann also davon ausgegangen werden, dass eine bestimmte "negative "Vorselektion insoweit besteht, als insbesondere schwere oder mit Komplikationen behaftete Fälle vornehmlich durch BG-Unfallkliniken versorgt werden. Hintergrund dafür ist deren technische, organisatorische und personelle Struktur.

Die Verlegung von Versicherten in BGUnfallkliniken verspricht bei der Behandlung Unfallverletzter erhebliche Vorteile, weil sowohl im Rahmen der Diagnostik als auch der Therapie die Ausstattung der 
entsprechenden Häuser dem modernsten Stand der Technik entspricht. Dies gilt in hervorzuhebender Weise für schwere Verletzungen wie die Fersenbeinfraktur. Die initiale Diagnosefindung bezüglich der Frakturform ist hier von wesentlicher Bedeutung (z. B. Frage der Gelenkbeteiligung oder des Dislokationsgrads). Hier können einerseits die Indikation zur operativen Versorgung sichergestellt und andererseits die operative Planung und Strategiefestlegung verletzungsbezogen optimiert werden.

Die Möglichkeit z. B. der multiplanaren Rekonstruktion nicht nur im Rahmen der präoperativen Vorbereitung, sondern auch als intraoperative Kontrolle bei der Versorgung von Fersenbeinfrakturen durch modernste Bildgebung, wie sie in einzelnen BG-Unfallkliniken Standard ist, kann von richtungsgebender Bedeutung für ein gutes Operationsergebnis und somit auch für eine optimierte Rehabilitationsphase nach operativer Versorgung sein.

Liegt eine bessere Erkenntnis über die Verletzungsform vor, kann gezielter eine optimale Therapieform gewählt werden. Die optimale Kontrolle des Therapieerfolgs mit multiplanarer Bildrekonstruktion sichert eine verbesserte Gelenkstellung und kann somit helfen, einer später einsetzenden posttraumatischen Arthrose entgegenzuwirken. Somit ist eine verbesserte Belastungsfähigkeit über eine längere Zeitdauer zu erwarten. Dieser Umstand kann positive Auswirkungen auf die Dauer der Arbeitsunfähigkeit, die Höhe der Minderung der Erwerbsfähigkeit und die Gesamtkosten der Heilbehandlung bewirken.

Die Annahme positiver Steuerungseffekte durch Verlegung in ein Kompetenzzentrum konnte durch die Datenanalyse gestützt werden. Die durchschnittlichen Gesamtkosten innerhalb der ersten 2 Jahre nach dem Arbeitsunfall waren $\mathrm{z}$. B. bei Fersenbeinfrakturen mit Komplikationen oder Nebendiagnosen in BG-Kliniken bei gleichzeitig kürzerer Arbeitsunfähigkeitsdauer nicht höher als in anderen Krankenhäusern (• Abb. 2, 3). Die frühe Einbeziehung eines Kompetenzzentrums erscheint damit wirtschaftlicher, als ein Trouble-Shooting nach misslungenen Vorbehandlungen in anderen Einrich-
Abb. 2 Vergleich der Behandlungs- und Rehabilitationskosten bei Frakturen des Fersenbeins mit Nebendiagnose

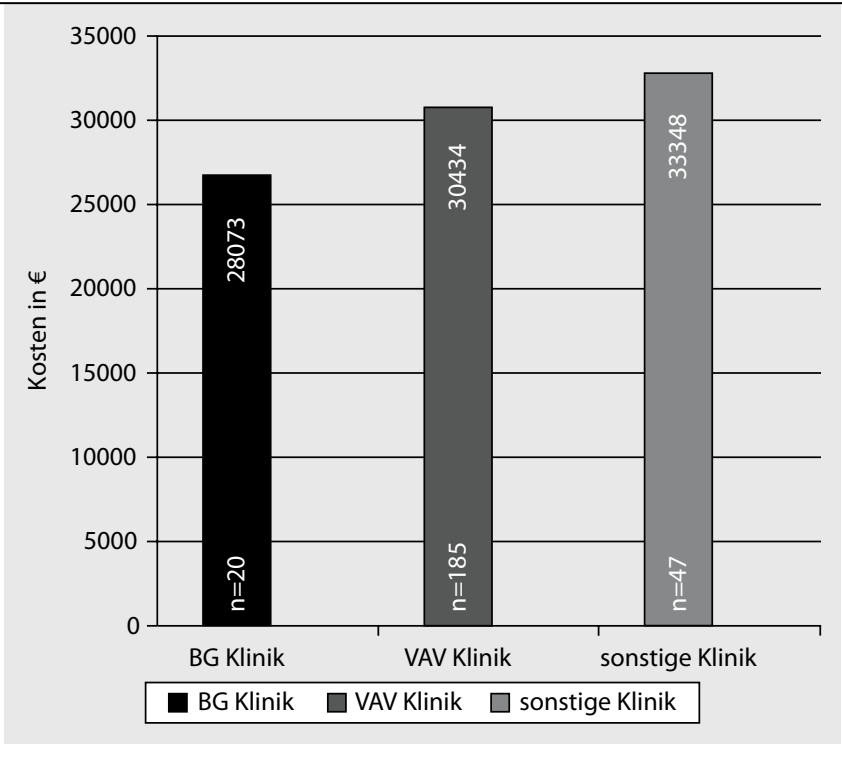

Abb. $3 \triangleright$ Vergleich der Arbeitsunfähigkeitsdauer (AU-Dauer) bei Frakturen des Fersenbeins mit Nebendiagnose

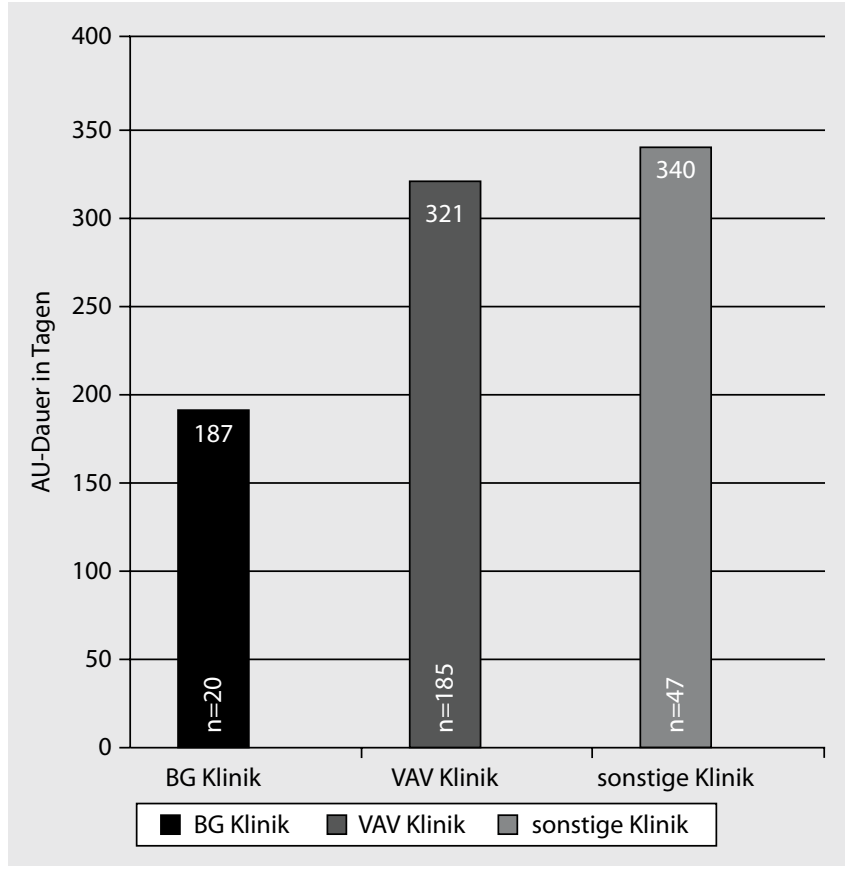

tungen zu betreiben. Ist die Behandlung durch weitere Nebendiagnosen erschwert oder treten Komplikationen im Heilverfahren auf, ist die volle Kompetenz des Behandlers gefordert. In diesen Fällen erweisen sich Kompetenzzentren mit der größtmöglichen Erfahrung als besonders geeignete Ansprechpartner. Ursächlich hierfür dürfte der Umstand sein, dass in diesen die Problemfallsteuerung gehäuft anfällt und durch die so gewonnene Erfahrung auch das Grundwissen über Lösungsstrategien für schwierige Fälle vorhanden ist. Von wesentlicher Bedeutung sind hier das frühzeitige Erkennen der Nebendiagnosen, die das Verfahren problematisch ge- stalten können, sowie ein sofortiges Gegensteuern bei Auftreten einer Komplikation.

Demgegenüber fiel auf, dass ein hoher Anteil an Fersenbein- und WeberC-Frakturen nicht in ein VAV-Krankenhaus oder eine BG-Unfallklinik verlegt, sondern in einer sonstigen Klinik versorgt wurde, obwohl diese Verletzungen auch im Zeitraum der betrachteten Unfälle Katalogverletzungen des Verletzungsartenverfahrens darstellten und damit eine unverzügliche Verlegung obligat war. Eine mögliche Fehlerquelle für dieses auf den ersten Blick alarmierende Ergebnis könnte der Umstand sein, dass nach 


\section{Benchmarking in der Gesetzlichen Unfallversicherung}
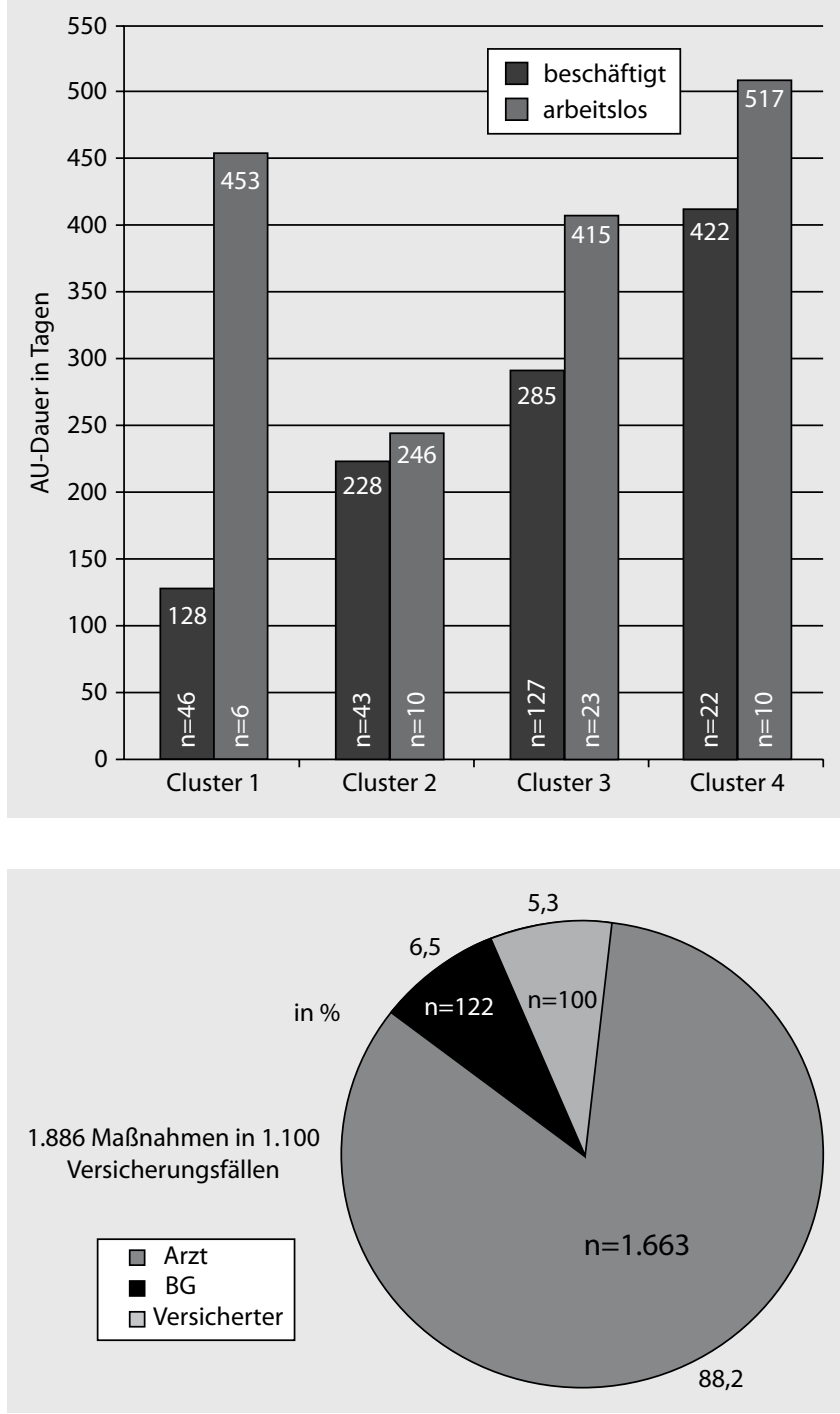

Abb. $4<$ Auswirkungen des Eintritts von Arbeitslosigkeit auf die AU-Dauer (Fersenbeinfraktur)

Abb. $5<$ Aktive Veranlasser von Interventionen im Rahmen der Heilbehandlung den aktuellen VAV-Listen die in den Akten als Behandler in der Akutphase angegebenen Kliniken zwar im Zeitpunkt der Behandlung zum VAV zugelassen waren, im Zeitpunkt der Auswertung aber keine VAV-Zulassung mehr besaßen. Andererseits bestand auch in der Arbeitsgruppe Einigkeit, dass zumindest in dem Untersuchungszeitraum, in dem die eingeschlossenen Verletzungen eingetreten waren, die Steuerung in den Verwaltungen der Benchmarkingteilnehmer auch insofern verbesserungswürdig war, als eine Verlegung trotz VAV-Katalog-Verletzung nicht in allen Fällen von den Sachbearbeitern mit dem nötigen Nachdruck verfolgt worden war.

\section{Resümee}

Als Best Practice zur Frage der Behandlerauswahl kann mithin empfohlen werden, schwere Verletzungen sowie sol- che mit Nebendiagnosen oder Komplikationen zeitnah gezielt zur Behandlung in eine BG-Unfallklinik zu steuern oder spätestens dorthin zu verlegen, wenn den Heilverlauf beeinträchtigende Umstände erkennbar werden, um das bestmögliche Heilergebnis zu erzielen. Der bekannte Effekt, dass Heilverfahrensverläufe besonders dann kostenaufwändig werden und Rentenfälle daraus entstehen, wenn eine BG-Unfallklinik erst am Ende einer nicht optimalen Serie von Behandlungsversuchen anderer Leistungserbringer als „Reparaturbetrieb“ von den Berufsgenossenschaften in Anspruch genommen wird, unterstreicht die formulierte Empfehlung in besonderer Weise [7]. Der Aufwand höherer Kosten für die Heilbehandlung rechtfertigt sich dann, wenn dadurch eine Qualität sichergestellt werden kann, aus der vergleichsweise niedrigere $\mathrm{Ge}$ samtfallkosten resultieren. Bei rechtzei- tiger und verantwortungsvoller Heilverfahrenssteuerung ließe sich hierdurch in einer erheblichen Zahl von Fällen ein medizinisch besseres und auch wirtschaftlicheres Heilverfahrensergebnis erzielen.

\section{Einfluss von Arbeitslosigkeit auf die Dauer der Arbeitsunfähigkeit}

Die Erhaltung des Arbeitsplatzes stellt einen weiteren Erfolgsfaktor der berufsgenossenschaftlichen Rehabilitation dar. Der Eintritt von Arbeitslosigkeit ist hingegen ein erheblicher Störfaktor.

\section{Resultat}

Welche Auswirkungen der Verlust des Arbeitsplatzes auf die Dauer der Arbeitsunfähigkeit (AU) haben kann, zeigte sich mit aller Deutlichkeit in der Datenanalyse. In Fällen, in denen der Versicherte seine Arbeit verlor, stieg die Dauer seiner Arbeitsunfähigkeit unabhängig vom Cluster bis auf das Doppelte an (• Abb. 4). Interessanterweise ließ sich die Verlängerung der Arbeitsunfähigkeitsdauer nach Eintritt der Arbeitslosigkeit - auch die umgekehrte Reihenfolge wäre denkbar gewesen - anhand der Aktenauswertung und der Erfahrung aus der Praxis belegen.

Die grundsätzlich zu berücksichtigende Tatsache, dass die Dauer der Arbeitsunfähigkeit aufgrund der Art der Arbeit (geistig/körperlich) und der unterschiedlichen Anforderungen durch Produktionsverfahren und Arbeitsorganisation der verschiedenen beteiligten Gewerbezweige erheblich differieren kann, wirkte sich im Untersuchungsergebnis nicht erkennbar aus.

Die zeitnahe Einbindung des Arbeitgebers in die Überlegungen zur beruflichen Rehabilitation des Versicherten im Unfallbetrieb kann dessen soziales Verantwortungsbewusstsein aktivieren und seine Mitwirkung fördern, sodass eine Kündigung des Versicherten vermieden werden kann. Denkbar sind hier:

- das frühzeitige Anbieten einer Eingliederungshilfe,

- die Vereinbarung einer Umsetzung oder

- eine betriebliche Qualifizierungsmaßnahme für eine andersartige Tätigkeit im Unternehmen. 
Wichtig ist in diesem Zusammenhang auch das Lebensalter des Versicherten. Tendenziell waren jüngere Arbeitnehmer mit kürzerer Beschäftigungsdauer und ältere Arbeitnehmer ab 49 Jahren überproportional höher von einer Kündigung bedroht als andere Altersgruppen.

\section{Resümee}

Als Best Practice muss die unverzügliche Einschaltung der Berufshilfe bzw. des Reha-Managers bei erkennbarer Verzögerung des Heilverfahrens abgeleitet werden. Von wesentlicher Bedeutung sind dabei die möglichst frühzeitige Einschätzung des Verletzungsausmaßes, des individuellen Belastungsprofils und der Reintegrationsfähigkeit des Versicherten an seinem originären Arbeitsplatz. Dabei sollten bereits in einem sehr frühen Stadium im Einvernehmen mit dem Versicherten und dem Arbeitgeber alle Möglichkeiten einer Erhaltung des Arbeitsplatzes erörtert werden. Oberste Priorität muss es sein, einem drohenden Arbeitsplatzverlust entgegenzuwirken und dem Risiko einer Verlängerung der Arbeitsunfähigkeitsdauer zu begegnen. Eine besondere Notwendigkeit zu diesem Vorgehen ergibt sich insbesondere bei körperlich anspruchsvollen, gering qualifizierten Tätigkeiten und schweren Verletzungen mit einer langen Arbeitsunfähigkeitsdauer, da diese Fälle ein hohes Komplikationspotenzial beinhalten.

\section{Aktive Heilverfahrenssteuerung}

Sie zeigt sich u. a. darin, ob die Sachbearbeiter eines Versicherungsträgers von sich aus prolongierte Heilverläufe erkennen, Maßnahmen wie EAP oder BGSW einleiten, eine Verlegung des Versicherten in ein Kompetenzzentrum oder einen Behandlerwechsel veranlassen oder beratende Ärzte einbinden. Der Unfallversicherungsträger kann und muss im Einzelfall steuernd in das Heilverfahren eingreifen, wenn die medizinische Betreuung des Versicherten nicht mehr dem Grundsatz „mit allen geeigneten Mitteln“ entspricht [8]. Die Unfallversicherungsträger können und dürfen sich weder nach der Rechtslage noch nach den Erfahrungen der Praxis blind auf das Funktionieren der medizinischen Rehabilitationskette verlassen.

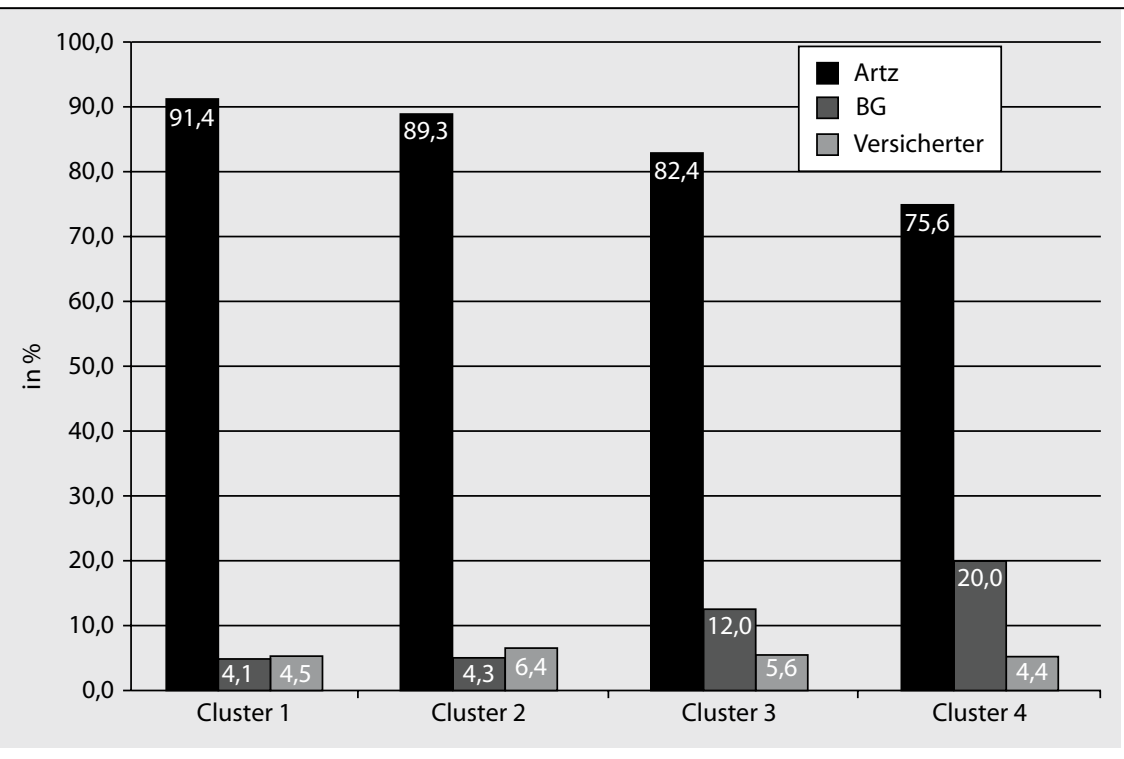

Abb. $6 \Delta$ Verteilung der Interventionen bezogen auf die einzelnen Cluster

Sie müssen vielmehr entsprechend ihrem weitergehenden Gesetzesauftrag der ihnen auferlegten Verantwortlichkeit für die Durchführung der Heilbehandlung selbst aktiv werden, um durch gezielte Überwachung und Steuerung des Heilverfahrens individuellen Bedürfnissen des konkreten Einzelfalls gerecht zu werden. ${ }^{1}$ Von großem Interesse war die Frage, ob sich hierbei ein unterschiedlicher Aktivitätsgrad der einzelnen Benchmarkingteilnehmer nachweisen lässt.

\section{Resultat}

Die Analyse der untersuchten Fälle erg$a b$, dass eine Steuerung des Heilverfahrens überwiegend durch die Ärzte erfolgte (• Abb. 5). Dies war zu erwarten, da insbesondere den D-Ärzten mit der Leitung des Heilverfahrens im medizinischen Bereich eine tragende Rolle zukommt [13]. Ihnen obliegt es, Versicherte zu beraten, zu behandeln sowie die am Heilungsprozess beteiligten Leistungserbringer zu koordinieren. Besonders deutlich wird die zentrale Steuerungsfunktion des D-Arztes bei der Entscheidung über eine Rehabilitationsmaßnahme (physikalische Therapie, EAP oder BGSW) oder bei der Einleitung einer Belastungserprobung [11].

\footnotetext{
1 Leuftink [10] forderte diesbezüglich ,Verantwortliches Verwaltungshandeln" und gab für die Umsetzung zahlreiche praktische Beispiele zu den grundlegenden Steuerungsmöglichkeiten der Verwaltung.
}

Im Ergebnis der weiteren Untersuchung ergab sich, dass die aktive Steuerung des Heilverfahrens durch die Träger der gesetzlichen Unfallversicherung in den einzelnen Clustern absteigend von Cluster 1 nach 4 zunahm. Mit dem zunehmenden Verbleiben von Unfallfolgen wurden also von den Benchmarkingteilnehmern ungünstige Heilverläufe erkannt und bei Verzögerungen auch aufgegriffen (• Abb. 6). Der Aktivitätsgrad differierte jedoch stark von Träger zu Träger. Obwohl die Kenntnis bei allen Teilnehmern unterstellt werden kann, dass ein Heilverlauf umso günstiger beeinflussbar ist, je früher eine Verzögerung des Heilverfahrens (z. B. aufgrund eintretender Komplikationen) erkannt und ihr entgegengewirkt wird, lässt die Analyse der Daten den Schluss zu, dass sich die Teilnehmer in ihrem Interventionsverhalten bezüglich des Erkennens von Versicherungsfällen mit verzögertem Heilverfahrensverlauf und entsprechender Reaktion durch ein Gegensteuern in starkem Maß voneinander unterscheiden. Für die Teilnehmer mit geringerem Aktivitätsgrad ergab sich hierbei ein erhebliches Potenzial für eine Verbesserung der Heilverfahrenssteuerung. Der Umstand, dass die teilnehmenden Unfallversicherungsträger einen Anteil von insgesamt lediglich $6,5 \%$ aller Steuerungsmaßnahmen auf sich vereinigen konnten, stellt insofern eine Standortbestimmung dar. $\mathrm{Ob}$ dieser Prozentsatz im $\mathrm{Zu}$ ge eines immer mehr zur Anwendung gelangenden Reha-Managements, bei dem 


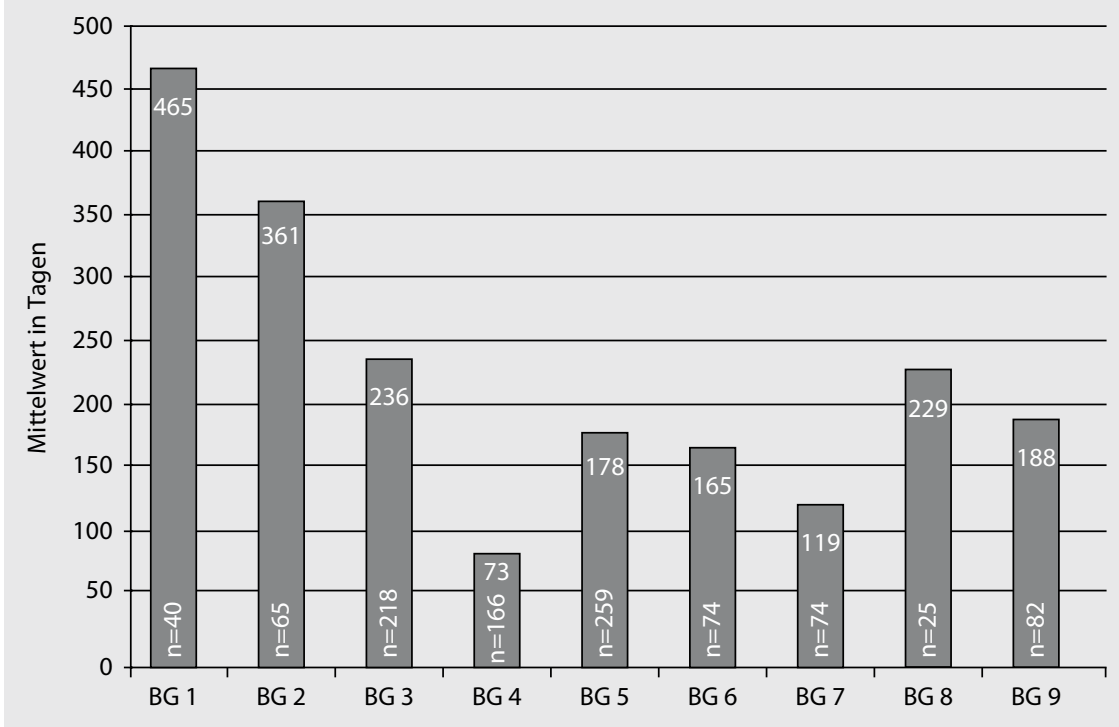

Abb. $7 \Delta$ Anonymisierter Vergleich der Laufzeit von Bescheiden (alle Verletzungen)

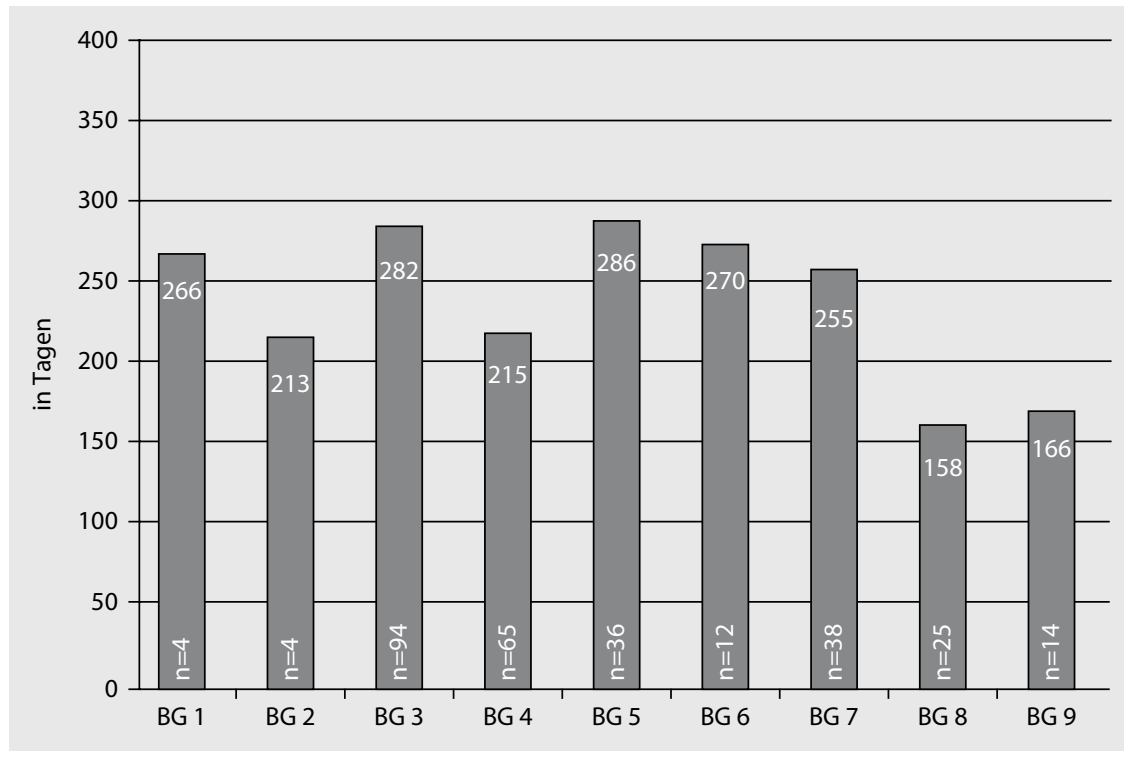

Abb. $8 \Delta$ Anonymisierter Vergleich der AU-Dauer bei Fersenbeinfraktur

der Unfallversicherungsträger Dreh- und Angelpunkt der Heilverfahrenssteuerung sein soll, steigerungsbedürftig ist, kann hier nicht beantwortet werden.

\section{Resümee}

Best Practice wäre ein frühzeitiges RehaManagement bei potenziell retardierten Heilverläufen, da die Teilnehmer mit frühzeitiger Steuerung von Problemfällen im Ranking der Heilverfahrensergebnisse die besseren Plätze für sich verbuchen konnten.

\section{Beispielhafte Darstellung differierender Heilverfahrensergebnisse}

In anonymisierter Weise lässt sich beispielhaft aufzeigen, wie unterschiedlich die Heilverfahrensergebnisse der Teilnehmer trotz Vergleichbarkeit der analysierten Unfallverletzungen sind.

\section{Laufzeit von Bescheiden}

Die Laufzeit, die ein Unfallversicherungsträger benötigt, um dem Versicherten einen Bescheid über die Anerkennung seines Arbeitsunfalls und ggf. über die Ge- währung von Leistungen zu übersenden, ist einer der Indikatoren dafür, wie effektiv eine Verwaltung arbeitet.

Die durchschnittliche Laufzeit für die Bearbeitung der 3 eingeschlossenen Unfallverletzungen zeigte unter den Teilnehmern eine erhebliche Spanne von $73 \mathrm{Ta}$ gen beim schnellsten Teilnehmer bis zu 465 Tagen ( Abb. 7). Bei den beiden Teilnehmern mit den kürzesten Laufzeiten galten Zielvereinbarungen zu anzustrebenden Durchschnittslaufzeiten von 2 bzw. 3 Monaten. Diese Zielvereinbarungen wurden u. a. durch frühzeitig und parallel eingeleitete Ermittlungen und Verfahrensschritte eingehalten.

\section{Arbeitsunfähigkeitsdauer bei Fersenbeinfrakturen}

Die durchschnittliche Arbeitsunfähigkeitsdauer nach Fersenbeinfrakturen reichte von 158-286 Tagen. Je nachdem, wie konsequent die Teilnehmer die oben genannten Steuerungsmöglichkeiten nutzten, konnten sie ein erheblich günstigeres Ergebnis im Vergleich zu anderen Trägern erzielen (• Abb. 8).

\section{Rehabilitationskosten bei Fersenbeinfrakturen}

Auch ein Blick auf die durchschnittlichen Kosten der medizinischen Rehabilitation lohnt einen Vergleich (• Abb. 9): Um ein optimales Heilverfahrensergebnis zu erreichen, musste trotz sehr vergleichbarer Verletzungen der erfolgreichste Teilnehmer Gesamtkosten von 8926 EUR innerhalb der ersten 2 Jahre nach dem Arbeitsunfall aufwenden, während der Teilnehmer mit dem ungünstigsten Ergebnis annährend den 3-fachen Betrag von 24.169 EUR zu leisten hatte. Auch dieser Vergleich weist zwischen dem ersten und dem letzten Platz ein erhebliches Optimierungspotenzial auf.

\section{Erhaltung des Arbeitsplatzes bei Fersenbeinfrakturen}

Sie glückte bei den erfolgreichsten Teilnehmern in $100 \%$ der Fälle, während die ungünstigste Quote bei 50\% lag (• Abb. 10). 


\section{Diskussion}

Das Projekt „Medizinische Rehabilitation" ergab, dass Benchmarking ein Weg ist, Optimierungspotenziale für die Heilverfahrenssteuerung aufzuzeigen. Relationen zwischen Kosten und Nutzen bzw. Kosten und Wirksamkeit sind im Gesundheitswesen zwar nicht leicht nachzuweisen, zumal bereits die Beschaffung vergleichbarer Daten Probleme bereitet. Dringend erforderlich wäre insofern eine bei allen Trägern einheitliche routinemäßige Qualitätssicherung für eine verbesserte Zielerreichung [11]. Hilfreich für die Bewertung von Steuerungsmaßnahmen und das Ablesen ihrer Effekte wäre zudem die Analysierbarkeit von Fallkontingenten anhand eines "data-warehouse“, da der Aufwand durch teils händisch ausgewertete Akten im Rahmen eines Benchmarkings zum einen in erheblichem Umfang Personalressourcen bindet und zum anderen eine hohe Fehleranfälligkeit birgt. Beides lieBe sich bei Einsatz von EDV auf dem aktuellem Stand der Technik, v. a. vor dem Hintergrund der in Zukunft regelmäßig zu erwartenden Benchmarkings, vermeiden. Je geringer die Zuverlässigkeit und Qualität der gewonnenen Daten sind, desto weniger lassen sich aus ihnen Schlussfolgerungen oder wirksame Maßnahmen zur Umsetzung von Erkenntnissen ableiten.

Hiervon unabhängig bleibt die Frage, ob die ideale Vorstellung rationalen Wirtschaftens auf reale Versorgungsprozesse in Rehabilitation und Teilhabe mit ihren individuellen Problemen und Kausalbeziehungen und der Abhängigkeit von der Entscheidungsmacht Dritter tatsächlich übertragen werden kann [9]. Die Ergebnisse von Heilverfahren werden zu einem guten Teil auch künftig von Faktoren beeinflusst werden, die einer Steuerung nicht zugänglich sind. Diese Umstände beeinflussen den Erkenntniswert von Benchmarking und mindern ihn.

Klare Kohärenzen zwischen Steuerungsmaßnahmen und Heilverfahrensergebnissen lassen sich oft genug nicht mit Fallauswertungen belegen, obwohl die Erfahrung dies vermuten lassen würde. Ein vorherbestimmbares Rehabilitationsergebnis im Sinne eines „GPS-Punkts“, auf das ein Heilverfahren mit zielgerichte-

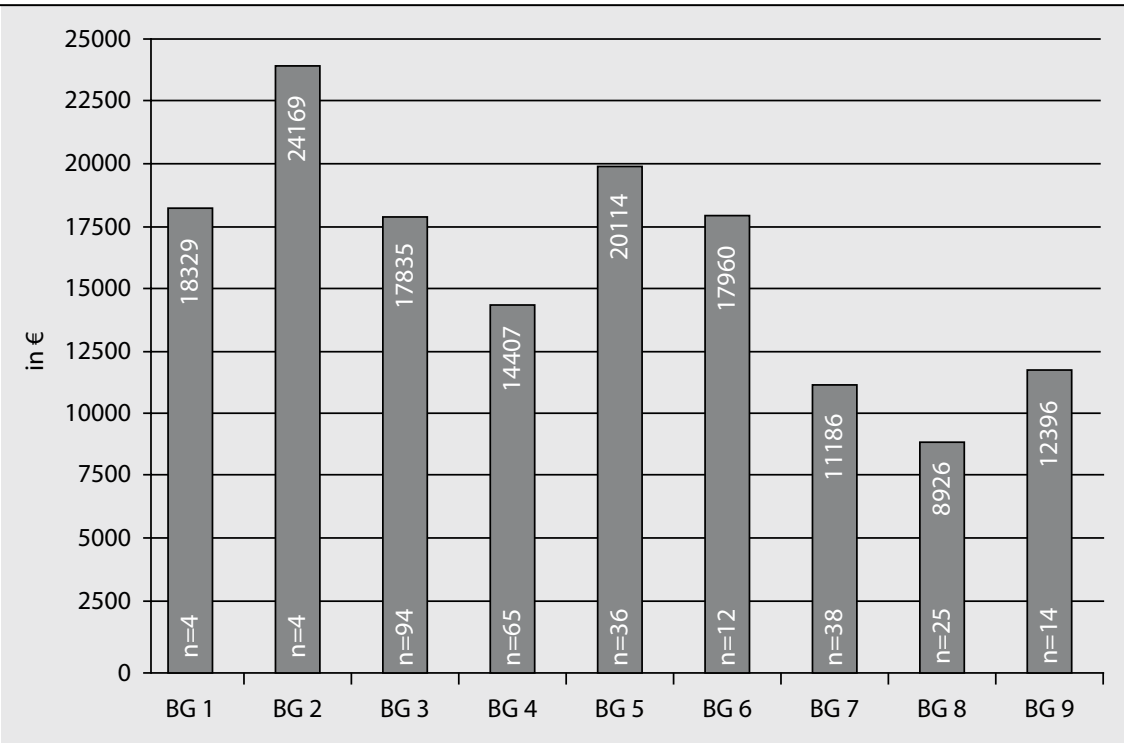

Abb. $9 \Delta$ Anonymisierter Vergleich der Behandlungs- und Rehabilitationskosten bei Fersenbeinfraktur

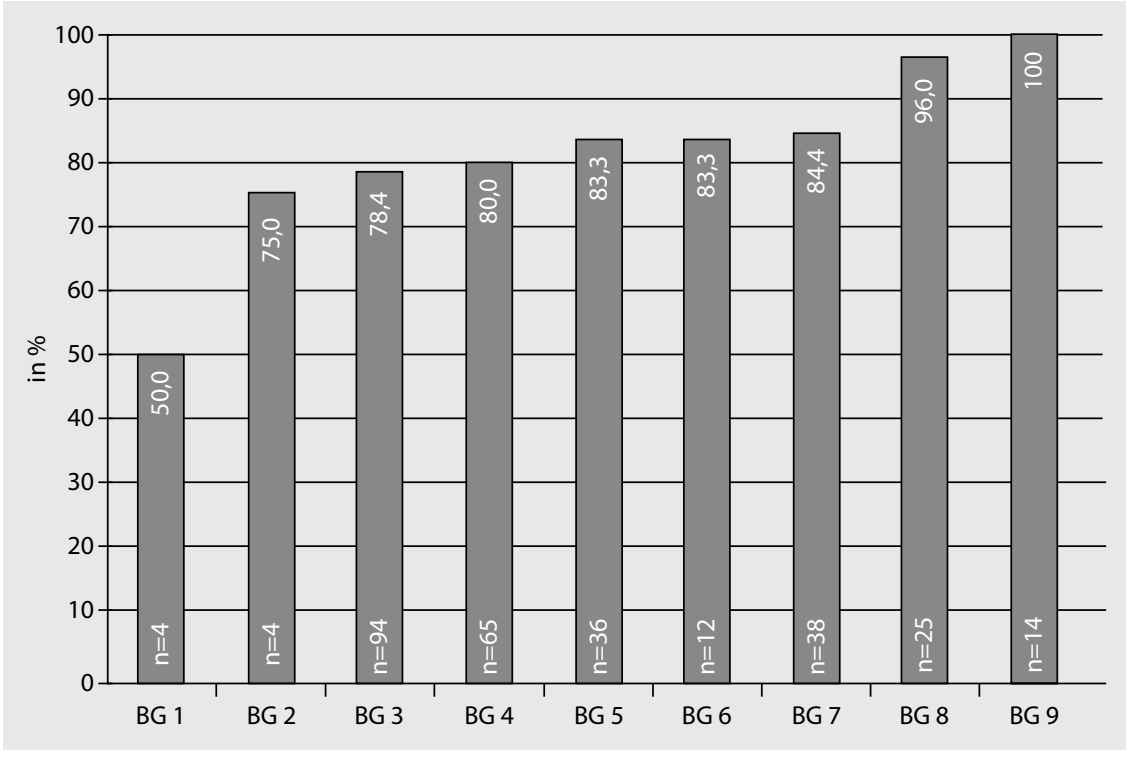

Abb. $10 \Delta$ Anonymisierter Vergleich der Arbeitsplatzerhaltungsquote bei Fersenbeinfraktur

ter Genauigkeit gesteuert werden könnte, wird es deswegen auch in Zukunft sicher nicht geben. Ein verlässlicher Schlüssel, ob und wie Versicherte möglichst schnell und nachhaltig, aber auch möglichst effizient wieder gesund und arbeitsfähig werden, objektiv gemessen werden kann, konnte für die gesetzliche Unfallversicherung bislang nicht entwickelt werden [2]. Des Weiteren kann ein Benchmarking im Gesundheitswesen nicht mit der gleichen Zuverlässigkeit Zusammenhänge zwischen Kosten und Nutzen aufweisen wie in einem Industrie- oder Handelsunternehmen. Neben den vielen steuerungs- unabhängigen Faktoren, die das Ergebnis des Heilverfahrens beeinflussen können, fehlt es an der exakten Vergleichbarkeit der Verletzungen, das Patientengut ist inhomogen (Unterschiede bezüglich Alter, körperliche oder geistige Tätigkeit, unterschiedliche Konjunktur der Gewerbezweige bezüglich Erhaltung des Arbeitsplatzes usw.), und die Anzahl der Fälle, die verglichen werden, ist zu gering, als dass statistischen oder wissenschaftlichen Ansprüchen Genüge getan werden kann.

Die Herstellung einer Maschine steht am Ende einer langen Reihe von Stellschrauben, die Einfluss auf den Kaufpreis 
haben. Die Kosten für zugelieferte Teile oder Rohstoffe, Lagerhaltung, Werbung und Personal gehören zu den wichtigsten Faktoren für ein konkurrenzfähiges Produkt. Eine Einflussnahme auf diese Variablen wirkt sich unmittelbar aus. Eine Analyse im Vergleich mit anderen Anbietern offenbart, auf welche der genannten Faktoren Einfluss genommen werden muss, um zu einem gleich günstigen Ergebnis zu gelangen. Die Kosten der medizinischen Rehabilitation eines Unfallverletzten sind dagegen nicht in vergleichbarer Weise beeinflussbar. Selbst identische Verletzungen bei gleichaltrigen Versicherten mit gleicher Tätigkeit können mit sehr unterschiedlichem Ergebnis ausheilen, ohne dass Fehler im medizinischen oder verwaltungsseitigen Handling des Falls ursächlich dafür sind. Hinzu kommen Unwägbarkeiten wie die subjektiv bedingte Schwankungsbreite bei der Einschätzung der Arbeitsunfähigkeitsdauer sowie der Minderung der Erwerbsfähigkeit zwischen Ärzten und Gutachtern.

Andererseits lässt sich nicht behaupten, für die messbare Qualität eines Heilverfahrens könnten keine nachprüfbaren Gesichtspunkte benannt werden. Dies liefe ebenso wie für andere Bereiche der öffentlichen Verwaltung auch für die gesetzliche Unfallversicherung auf eine Genietheorie im Sinne des Kunstverständnisses der Romantik hinaus, das für sich beansprucht, das Eigentliche der Kunst lasse sich nicht beschreiben, weil es eben genial und damit nicht greifbar sei [12]. Das Benchmarking hat - im Gegenteil gezeigt, dass bei aller gebotenen Vorsicht bezüglich der Interpretation seiner Ergebnisse Erfolgsfaktoren und Benchmarks für die Heilverfahrenssteuerung sehr wohl objektivierbar sind, was die Möglichkeit von Betriebsvergleichen zwischen den Trägern der gesetzlichen Unfallversicherung eröffnet. Dabei kommt der gesetzlichen Unfallversicherung im Gegensatz zu den Krankenkassen der Vorteil zugute, dass sie über sämtliche Informationen zu Leistungen und Kosten bezogen auf den einzelnen Versicherungsfall verfügen [2] und durch die Begutachtung zur Feststellung von Verletztenrenten in einer Vielzahl von Fällen auch das Ergebnis ihrer Rehabilitationsbemühungen in Gestalt verbleibender Unfallfolgen und einer diese bewertenden Minderung der Erwerbsfähigkeit sehen.

Diese Umstände machen die Qualität der medizinischen Rehabilitation anhand ihrer Ergebnisse messbar und vergleichbar. Alles was messbar ist, sollte letztlich auch einer Verbesserung zugänglich sein. Wenn auch die Methodik nach diesem ersten trägerübergreifenden Projekt, das in der gesetzlichen Unfallversicherung zum Themenkreis „Rehabilitation“ bisher durchgeführt wurde, sicher noch der Verfeinerung und Präzision bedarf, ist damit dennoch ein Grundstein gelegt. Nachhaltige Verbesserung setzt aber die regelmäßige Beteiligung an einem Benchmarking voraus, da ohne diesen Einsatz eine Standortbestimmung im Vergleich zu anderen Sozialversicherungsträgern nicht möglich ist. Wenn es gelänge, statt des Vergleichs von Durchschnittswerten valide verletzungsspezifische Referenzwerte für eine optimierte Heilverfahrenssteuerung zu ermitteln, würde dies den Stellenwert von Benchmarking für die gesetzliche Unfallversicherung erheblich steigern.

In welchem Umfang Benchmarking dazu beitragen kann, einen gezielteren Einsatz von Ressourcen zu ermöglichen und Effizienzpotenziale zu realisieren, ist gegenwärtig noch völlig offen. ${ }^{2}$ Ohne ein kontinuierliches Benchmarking sind jedoch eine Qualitätssicherung, eine Kostenkontrolle im Vergleich zu anderen Trägern und letztlich eine dauerhafte Optimierung der Heilverfahrenssteuerung künftig nicht mehr denkbar.

\footnotetext{
2 In ihrer Antwort auf die Kleine Anfrage der Abgeordneten Klaus Ernst, Dr. Martina Bunge, Werner Dreibus und weiterer Abgeordneter sowie der Fraktion „DIE LINKE“, wie die im Eckpunktepapier der Bund-Länder-Arbeitsgruppe [3] formulierte Vorgabe an die Berufsgenossenschaften zur Einsparung von 20\% der Verwaltungskosten in den ersten 5 Jahren nach Umsetzung der Reform organisatorisch realisiert werden solle, hat die Bundesregierung ursprünglich die Auffassung vertreten, einen wesentlichen Beitrag hierzu könne u. a. das für die gesetzliche Unfallversicherung künftig als obligat vorgesehene Benchmarking leisten [4]. Eine substantiierte Fundierung dieser Forderung, die ohne Differenzierung nach bisherigen Bemühungen und Erfolgen für alle Träger gleichermaßen gelten soll, lag dieser Einsparvorgabe, soweit ersichtlich, nicht zugrunde
}

\section{Korrespondenzadresse}

\section{Behrens}

Berufsgenossenschaft Chemie, Stolberger Straße 86, 50933 Köln

kkuehnel@bgchemie.de

Interessenkonflikt. Keine Angaben

\section{Literatur}

1. Behrens M, Bergschmidt S, Jürs E et al. (2007) Benchmarking-Projekte in der gesetzlichen Unfallversicherung. Trauma Berufskrankh 9: 133

2. Breuer J, Mehrhoff F (2001) Management von Personenschäden - Herausforderungen und Strategien aus der Sicht der gesetzlichen Unfallversicherung. Die BG 2001: 13

3. Bund-Länder-Arbeitsgruppe (2006) Eckpunktepapier. Ausschussdrucksache 16(11)340. Deutscher Bundestag, Berlin

4. Bundesregierung (2006) Drucksache 16/5536. Deutscher Bundestag, Berlin

5. Fischer M (1996) Die Wechselwirkungen zwischen der gesetzlichen Unfallversicherung und der Chirurgie. HVBG, St Augustin, S 27

6. Fornoff C, Schmickal T, Ritter F (2006) Rehabilitationsmanagement. Trauma Berufskrankh [Suppl 1] 8: S93-S104

7. Förster B (2001) Zur Rehabilitation Arbeitsunfallverletzter in Sonderstationen. Die BG 2001: 86

8. Hauck K, Keller W, Noftz W et al. (2005) Sozialgesetzbuch VII: Gesetzliche Unfallversicherung, Kommentar. Schmidt, Berlin, §26 SGB VII Rn.48

9. Hehling W (2003) Die gemeinsame Empfehlung "Qualitätssicherung" nach §20 Sozialgesetzbuch (SGB) IX. Die BG 2003: 514

10. Leuftink D (1991) Die Überwachung und Steuerung des Heilverfahrens aus Sicht der Verwaltung. Die BG 1991: 665

11. Mehrhoff F, Weber-Falkensammer H (2000) Qualität und Wirtschaftlichkeit der Leistungen zur Heilbehandlung und Rehabilitation in der gesetzlichen Unfallversicherung. Die BG 2000: 104

12. Röhl K (1999) Justiz als Wirtschaftsunternehmen? Budgetierung, Controlling und Professionalisierung der Justizverwaltung, Vortrag für den Deutschen Richtertag in Karlsruhe am 04.10.1999, S 13

13. Weller S (1991) Die Überwachung und Steuerung des Heilverfahrens aus ärztlicher Sicht. Die BG 91: 663-664 